\title{
RECIPROCAL SPACE MAPPING OF EPITAXIAL MATERIALS USING POSITION-SENSITIVE X-RAY DETECTION
}

\author{
S.R. Lee, B.L. Doyle, T.J. Drummond, J.W. Medernach and R.P. Schneider, Jr. \\ Sandia National Laboratories, Albuquerque, NM, 87185-1056
}

\begin{abstract}
Reciprocal space mapping can be efficiently carried out using a position-sensitive $\mathrm{x}$ ray detector (PSD) coupled to a traditional double-axis diffractometer. The PSD offers parallel measurement of the total scattering angle of all diffracted $\mathrm{x}$-rays during a single rocking-curve scan. As a result, a two-dimensional reciprocal space map can be made in a very short time similar to that of a one-dimensional rocking-curve scan. Fast, efficient reciprocal space mapping offers numerous routine advantages to the x-ray diffraction analyst. Some of these advantages are the explicit differentiation of lattice strain from crystal orientation effects in strain-relaxed heteroepitaxial layers; the nondestructive characterization of the size, shape and orientation of nanocrystalline domains in orderedalloy epilayers; and the ability to measure the average size and shape of voids in porous epilayers. Here, the PSD-based diffractometer is described, and specific examples clearly illustrating the advantages of complete reciprocal space analysis are presented.
\end{abstract}

\section{INTRODUCTION}

Recently, reciprocal space mapping using $\mathrm{x}$-ray diffraction has been enjoying growing popularity as an analytical tool for the analysis of epitaxial semiconductor materials. A partial list of topics of current interest includes reciprocal space analysis of: the onset and evolution of strain relaxation in both group II-VI and III-V heteroepitaxial systems [1,2], the strain state and grating structure of two-dimensional periodic arrays of semiconductor pillars [3], strain and damage in ion-implanted semiconductors [4] and defect precipitation in as-grown and annealed homoepitaxial layers [5]. The increasing application of reciprocal space analysis by $\mathrm{x}$-ray diffraction is probably a result of both the recent advent of commercially-available, high-quality, turn-key, triple-axis diffractometers, and the associated emerging realization that a complete analysis of reciprocal space by $\mathrm{x}$ ray diffraction often provides far superior information about the material of interest.

Until recently, reciprocal space mapping of semiconductor epitaxial layers by $\mathrm{x}$-ray diffraction has been carried out almost exclusively using triple-axis diffractometers. These

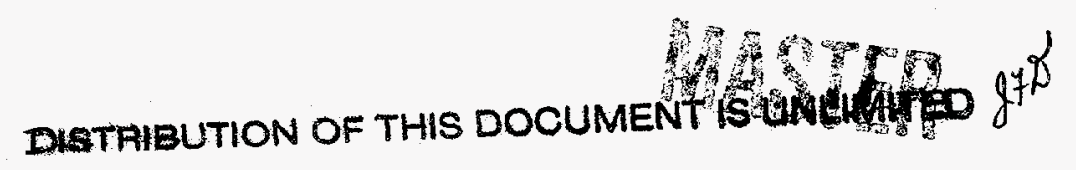




\section{DISCLAIMER}

Portions of this document may be illegible in electronic image products. Images are produced from the best available original document. 
instruments mechanically rock the second axis containing the sample through a range of incident $\mathrm{x}$-ray angles. At each rocking angle an additional serial scan of an analyzer crystal mounted on the third axis is performed. The analyzer crystal measures the scattering angle of the diffracted $\mathrm{x}$-rays emerging from the sample crystal. Precise measurement of both the rocking angle and the scattering angle affords the triple-axis diffractometer with its principle advantage -- the ability to map reciprocal space with extremely high resolution. The disadvantage of the triple-axis technique is the long time required to acquire the multidimensional reciprocal space data. The long data collection time arises because of the purely serial nature of the two-dimensional mechanical scan of reciprocal space.

This difficulty may be overcome using an approach perhaps first suggested by Mathieson [6]: Rather than using a mechanically scanned analyzer crystal, or slit, a position-sensitive $\mathrm{x}$-ray detector (PSD) is added to a rocking curve system in order to measure the scattering angle of the diffracted $x$-rays. In contrast to a triple-axis scheme, the PSD measures the total scattering angle of all diffracted $x$-rays in a parallel fashion. This allows simultaneous probing of multiple regions of reciprocal space. The net effect of using a PSD is that a complete reciprocal space map can be made in a time similar to that required to perform a typical rocking curve analysis.

A simple diffractometer developed in recent years by Thompson, Doyle et al. [7] which uses a PSD to make reciprocal space maps of epitaxial materials is presented here. In essence, the system consists of a resistive-curved-wire, position-sensitive, proportional counter coupled to a conventional double-axis diffractometer. The present treatment includes a fuller discussion of factors affecting the resolution and utility of the instrument. The advantage of high speed reciprocal space mapping obtained through the use of positionsensitive detection does entail certain disadvantages: Limitations imposed on the reciprocal space resolution, and the maximum diffracted beam intensity, by the use of a curved-wire proportional counter are discussed.

This diffractometer has now been extensively applied to a much wider variety of materials analysis problems than when it was first developed, and we have selected some examples for presentation which illustrate the power of reciprocal space mapping using position-sensitive x-ray detection. Some of the examples reemphasize the importance of previously recognized benefits of reciprocal space mapping (for example, explicit separation of lattice strain and crystal orientation effects), while others suggest alternative uses for reciprocal space mapping that have not been as fully explored (for example, the detailed characterization of the size, shape and orientation of nano-crystalline structures and domains present in some epitaxial layers). These examples include: the rapid and accurate measurement of strain distributions, composition, mosaic structure and average layer orientation in highly strain-relaxed, $\operatorname{In}_{x}(\mathrm{AlGa})_{1-\mathrm{x}} \mathrm{As}$ ( $\left.\mathrm{x}=0.04-0.4\right)$, step-graded, buffer layers; the characterization of the size, shape and orientation of ordered-alloy domains $\mathrm{In}_{0.48} \mathrm{Ga}_{0.52} \mathrm{P}$ epilayers; and the measurement of the void size and shape in porous $\mathrm{Si}$ films. 


\section{DIFFRACTOMETRY USING POSITION-SENSITIVE X-RAY DETECTION}

\section{The Basic Apparatus:}

The apparatus that Thompson and Doyle developed for reciprocal space mapping is shown schematically in Figure 1. The main elements of the system are a sealed-tube x-ray generator, a double-axis goniometer, a curvedwire proportional counter and a CAMACbased data acquisition and control system. The $\mathrm{x}$-ray generator produces $\mathrm{Cu} \mathrm{k}_{\alpha}$ radiation using a $1.5 \mathrm{~kW}$, fine-focus, long-anode $\mathrm{x}$-ray tube. The tube is oriented so that the $\mathrm{x}$-ray source has an apparent width and height of 40 $\mu \mathrm{m} \times 8 \mathrm{~mm}$. A monochromatic $\mathrm{Cu} \mathrm{k}_{\alpha 1} \mathrm{x}$-ray beam is produced by a one-bounce, (004), $\mathrm{Si}$ single-crystal with collimators placed before and after. The second collimator serves to remove the $\mathrm{Cu} \mathrm{k}_{\alpha 2}$ line from the conditioned beam. The angular divergence, $\alpha$, of the $x$-ray beam delivered by this system is $40^{\prime \prime}$ fullwidth at half maximum (FWHM). Since the monochromator and second crystal are in a (+-

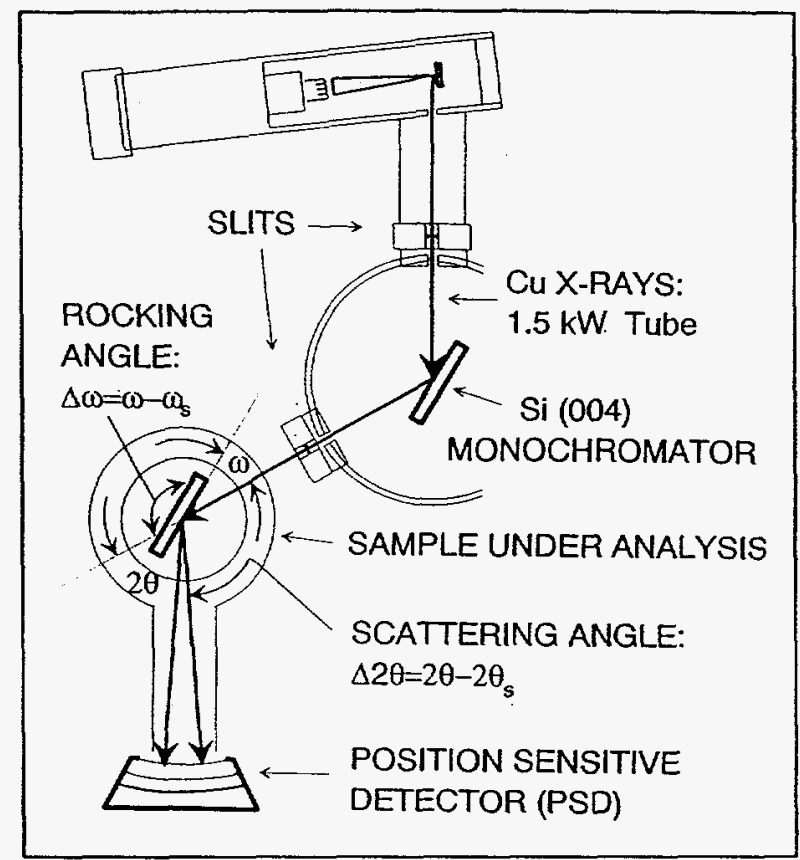

Figure 1. Schematic diagram of a double-axis diffractometer with a curved-wire proportional counter for position-sensitive detection of diffracted $\mathrm{x}$-rays.

) arrangement, the system is nondispersive for (004) Si and has a variable dispersion and rocking curve width for other sample crystals and orientations (from 14" for (224) Si to 190" for (008) InSb). The crystal under analysis is rocked with up to 1 " resolution using a stepping motor under control of the CAMAC system. The maximum automated rocking angle range is 5.6 degrees.

The novel and important aspect of the apparatus is the use of the curved-wire proportional counter in place of a standard scintillation counter. The curved-wire counter is mounted on a manually positioned $2 \theta$ axis , and provides position-sensitive $x$-ray detection over an arc $50 \mathrm{~mm}$ in length on a $250 \mathrm{~mm}$ radius of curvature. This arrangement enables position-sensitive, parallel detection of all x-rays within a total $2 \theta$ angular range of $11.5^{\circ}$. In this system, the detector is stationary during the reciprocal space scan. The curved-wire is used to maintain the incident x-ray path normal to the detector wire along its entire length. This reduces path-length-induced broadening of the detector's position resolution due the 6-mm-deep sensitive volume of the detector. The height of the detector sensitive volume is $10 \mathrm{~mm}$, which is well-matched to the maximum vertical extent of the diffracted $\mathrm{x}$ ray beam. The detector uses a 0.15 liter/hour flow of P10 gas at 6.6 bars, and the detector entrance window is 0.4 -mm-thick beryllium. The detection efficiency for $\mathrm{Cu}_{\alpha} \mathrm{x}$-rays using this detector configuration is $62 \%$ when measured relative to a NaI scintillation counter. 
The spatial resolution for the detector was measured to be $120 \mu \mathrm{m}$ FWHM using a beam collimated with a $30 \mu \mathrm{m}$ wide slit.

As in triple-axis diffractometry, measurement of both the rocking angle, $\omega$, of the sample and the total scattering angle, $2 \theta$, of diffracted $x$-rays gives the diffractometer the capability to produce a two-dimensional map of diffracted x-ray intensity as a function of position in reciprocal space. Relatively simple equations, which directly transform diffracted intensity versus $\omega$ and $2 \theta$ to diffracted intensity versus reciprocal space coordinates, can be derived using an Ewald-Sphere construction. These equations, which appear in reference [8], were used to calculate the reciprocal space maps to be presented below.

A reciprocal space map is made by collecting an $\mathrm{NxM}$ array of intensity values, where $N$ is the number of angular steps in $\omega$, and $M$ is the number of angular steps in $2 \theta$, needed to completely a map of a localized region of reciprocal space. Typical values for $N$ and $\mathrm{M}$ are -100 or more. Thus, a triple-axis diffractometer, which must serially scan both $\omega$ and $2 \theta$, will require a time of $-100 \times 100 \times \Delta t$ to acquire a reciprocal space map. In marked contrast, a PSD-based system, which only has to scan $\omega$, requires a time of only $-100 \mathrm{x} \Delta \mathrm{t}$. In our first-generation apparatus, the tremendous time savings gained by taking advantage of the parallelism of PSD-based detection does entail certain important tradeoffs. Some of the disadvantages which arise are solely due to the use of the positionbased measurement of total scattering angle, while others result from our particular choice to use a curved-wire gas-filled proportional counter as the PSD.

\section{System Resolution in Angular Space and Reciprocal Space:}

The most important trade-off that is made with the present apparatus is that the $2 \theta$ resolution is approximately ten-times poorer than in a typical triple-axis system employing a Ge (220) monochromator and analyzer crystal. Since the $\omega$ resolution is not effected by use of the PSD, the angular resolution function of the present PSD-based system becomes a highly asymmetric ellipse upon transformation to reciprocal space. This elliptically shaped response has a minor axis diameter of $-1.5 \times 10^{-4} \AA^{-1}$ FWHM (for nondispersive analysis), that is primarily determined by the system's $\omega$ angular resolution, and a major axis diameter of $-2.6 \times 10^{-3} \AA^{-1}$ FWHM, that is primarily determined by the system's $2 \theta$ resolution. By comparison, the resolution function of the above triple-axis system is more nearly circular in reciprocal space with a diameter of $-2.8 \times 10^{-4} \AA^{-1} \mathrm{FWHM}$. Since the most fundamental limitation of our apparatus is the poorer $2 \theta$ resolution, and the corresponding decrease in reciprocal space resolution, some important questions are: What determines the $2 \theta$ resolution in a PSD-based system? What are the prospects for improvement?

To first order, the $2 \theta$ angular resolution is determined by the system's overall position resolution, $w$, divided by the distance, $r_{d}$, the PSD is placed from the sample crystal. The overall position resolution has two basic contributions: The first is the position resolution of the PSD, $w_{p}$, and the second is the width of the beam as it arrives at the PSD, $w_{d}$. The width of the beam at the PSD in turn depends on the initial width of the 
beam at the source, $w_{0}$, and the subsequent expansion of the beam along the path from the $\mathrm{x}$-ray source to the sample, $r_{S}$, and along the path from the sample to the PSD. Finally, the rate of beam width expansion depends on the divergence, $\alpha$, of the beam produced by the system's beam conditioner. The basic geometrical considerations determining the $2 \theta$ resolution are summarized in Figure 2. Adding in quadrature the nominal angular widths associated with: (1) the finite initial beam width, $\Gamma_{a}$, (2) beam width expansion due to the angular divergence of the conditioned $\mathrm{x}$ ray source, $\Gamma_{b}$, and (3) the PSD angular resolution, $\Gamma_{c}$, we obtain the approximate FWHM of the $2 \theta$ resolution function, $\Gamma_{2 \theta}$ :

$$
\begin{aligned}
\Gamma_{2 \theta} & =w / r_{d} \\
& =\left[\left(w_{o}{ }^{2}+\alpha^{2}\left(r_{s}+r_{d}\right)^{2}+w_{p}{ }^{2}\right) 1 / 2\right] / r_{d} \\
& \left.=\left(\Gamma_{a}{ }^{2}+\Gamma_{b}{ }^{2}+\Gamma_{c}\right)^{2}\right)^{1 / 2} .
\end{aligned}
$$

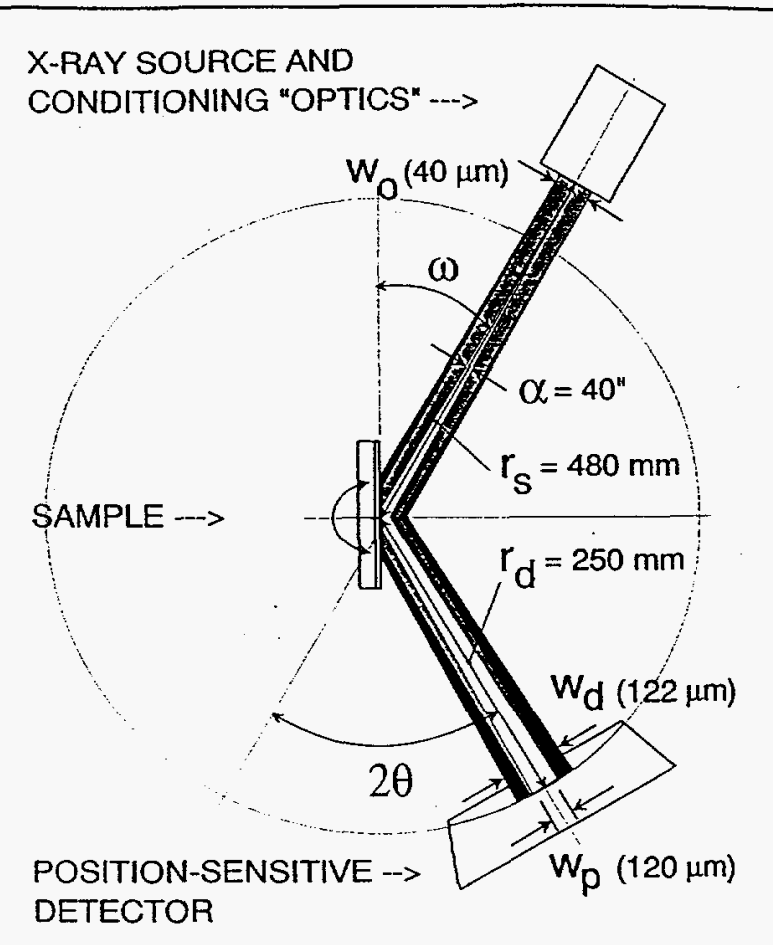

Figure 2. The geometrical factors which determine the $2 \theta$ resolution of a diffractometer which uses a PSD.

For the present system, $\Gamma_{\mathrm{a}}=33^{\prime \prime}, \Gamma_{\mathrm{b}}=117^{\prime \prime}$, and $\Gamma_{\mathrm{c}}=100^{\prime}$. These factors result in a combined theoretical resolution of $\Gamma_{2}=160 "$. For comparison, a measurement of the $2 \theta$ resolution made using a Si sample set for nondispersive (004) diffraction is shown in Figure 3. The measured FWHM of 160" agrees with our analysis.

It should be noted that the above analysis assumes symmetrical diffraction at the sample. The use of asymmetric diffraction planes will modify the final beam width at the PSD. Grazing-exit geometries will compress the beam width and increase the beam divergence. Conversely, grazing-incidence geometries will expand the beam width and decrease the beam divergence. The change in beam width due to geometrical compression or expansion at the sample tends to dominates over the path-length-induced change in width due to the change in beam divergence at the sample exit. Thus, it is generally preferable to use a grazing-exit sample geometry for asymmetric diffraction planes in order to

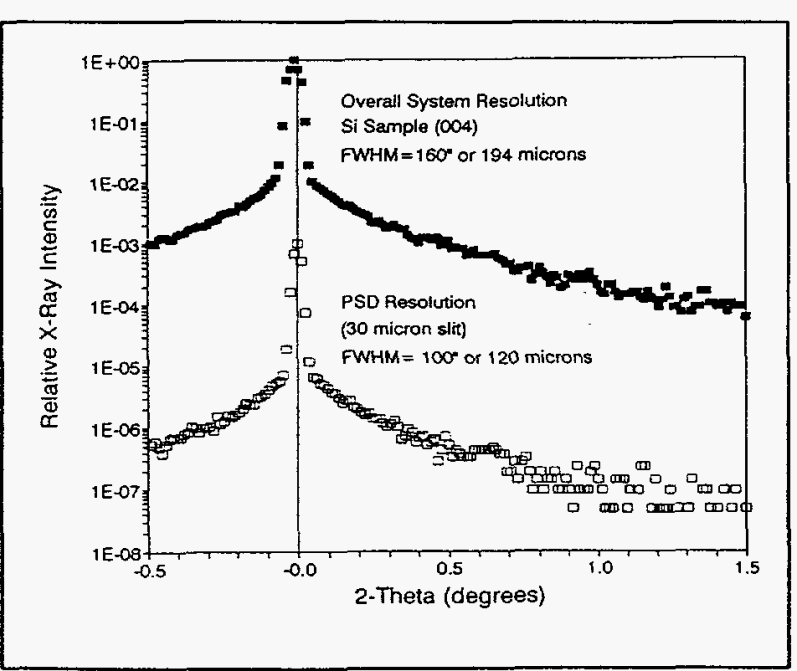

Figure 3. The overall $2 \theta$ resolution of the present PSD-based diffractometer, and the resolution of the PSD when collimated with a $30-\mu \mathrm{m}$-wide slit. 
preserve the $2 \theta$ resolution. This is in contrast to the more usual practice of using grazingincidence scattering for high resolution rocking curve and triple-axis analyses.

Using Equation (1), one can immediately begin to discern the parameters which must be optimized in order to improve the resolution of our first-generation device. For example, simple improvements that could be made would be to install a Ge (220), 4-bounce monochromator and obtain a detector that could be operated at twice the distance from the sample. This would reduce $\Gamma_{\mathrm{a}}$ to $17^{\prime \prime}, \Gamma_{\mathrm{b}}$ to $24^{\prime \prime}$ and $\Gamma_{\mathrm{c}}$ to $50^{\prime \prime}$, and consequently, would reduce $\Gamma_{2 \theta}$ to 58 ". Of course, the intensity tradeoff due to use of the high resolution monochromator would be a rather large reduction from $2 \times 10^{6}$ to $2 \times 10^{5}$ photons/sec. Improvement of the $2 \theta$ resolution to below $58^{\prime \prime}$ would require more extensive modifications, but is entirely possible. Higher spatial resolution detectors, larger detector distances, more compact beam-conditioning optics located extremely near the sample, and advanced optics which employ asymmetrically cut crystals and/or microfocus $x$-ray sources to further decrease the beam width, are all themes which could be explored in various combinations to improve $2 \theta$ resolution.

One final aspect of the $2 \theta$ resolution which we have not touched on is the extended low-intensity tailing of the $2 \theta$ response shown in Figure 3. The maximum tail amplitude is $-1.1 \times 10^{-2}$ times that of the main peak amplitude and falls to $-1 \times 10^{-4}$ at $1.5^{\circ}$ from the main peak. This tailing of the $2 \theta$ resolution function gives rise to a reciprocal space feature which is analogous to the so-called "analyzer streak" in a conventional triple-axis system. As shown in Figure 3, when a collimating slit is placed between the sample and detector, the area under the tails remains a constant fraction of the main peak area $(-10 \%)$. Thus, the primary mechanism producing the tails in the response of the gas-filled curved-wire proportional counter arises within the detector, or the detector electronics, and is not due to external factors such as scattering along the beam path. We have not yet unambiguously determined the specific source of the tailed response.

\section{Detector Dynamic Range Limitations:}

An important practical disadvantage of curved-wire proportional counters relative to ordinary scintillation counters is their non-robust nature. The detector contains a $25-\mu \mathrm{m}$ diameter, carbon-coated quartz wire. The maximum point-loaded count rate of the detector wire required to avoid thermal damage is $-10,000 \mathrm{cps} / \mathrm{mm}$ [9]. Since the width of the diffracted beam at the detector is $194 \mu \mathrm{m}$ FWHM for symmetric (004) diffraction from $\mathrm{Si}$, the maximum count rate near sharp diffraction features must be less than $-2100 \mathrm{cps}$ in the present system to avoid wire damage due to point loading. Compression of the beam width by highly asymmetric, grazing-exit diffraction at the sample lowers this limit further. In a conventional triple-axis system the detector is very robust and has a high dynamic range ($\left.10^{-1}-10^{6} \mathrm{cps}\right)$. One way to partially approximate this dynamic range using a curved-wire proportional counter is to reduce the beam intensity incident on the counter in instances, or at positions, where point-loading damage may result. This may be done using manual or 
automated scanning of the diffractometer power, or by precision placement of narrow-width attenuator foils at the $2 \theta$ position of the substrate peak.

\section{THE ADVANTAGES OF RECIPROCAL SPACE ANALYSIS USING A PSD}

In the above discussion we have tried to show that reciprocal space maps of epitaxial materials can be made with unprecedented speed $(-100 \mathrm{x}$ faster than is customary) using position-sensitive $\mathrm{x}$-ray detection, but the important tradeoffs for our first-generation apparatus are loss of resolution $(-10 \mathrm{x}$ poorer than a typical triple-axis) and decreased dynamic range (in the worst case, up to $-1000 \mathrm{x}$ poorer). Given this new capability, which has different strengths and weaknesses in comparison with conventional triple-axis analysis, a question which naturally arises is: What types of materials and structures are well-suited for analysis by this technique, and what types are not?

The samples that tend to be less attractive candidates for analysis are highly perfect structures, which tend to require both high dynamic range and excellent resolution in order to get the best results. Diffraction from this class of structures often produces a onedimensional modulation in reciprocal space. In this instance, the reciprocal space of the structure is most efficiently mapped using either a rocking curve or a triple-axis line scan, and a full two-dimensional map is not necessarily fruitful. Certain types of analytical problems, for example, the analysis of small amounts of misorientation, or so-called mosaic, in nearly perfect substrates or in nearly perfect thick epilayers, or the analysis of diffuse scattering due to point defects in regions of reciprocal space extremely close to the substrate position, are not the forte of the present device. These types of analyses are still best done with a triple-axis instrument.

The samples that are best analyzed using the present diffractometer are more often those which are less perfect in some sense, or those which are relatively perfect but are very thin. Samples of this type will tend to have, either larger, or unusual multidimensional features in reciprocal space, will tend to require only moderate dynamic range for analysis, and will tend to have inherently lower x-ray reflectivity. Consequently, they are often well-matched to our technique. Below, three prototypical applications of PSD-based reciprocal space mapping are given which illustrate the importance and usefulness of fast, efficient reciprocal space analysis at moderate resolution.

\section{The Explicit Differentiation of Lattice Strain from Crystal Orientation Effects:}

One of the earliest applications of triple-axis reciprocal space mapping was to study lattice-parameter and orientation variations in single crystals [10]. The analysis of lattice strain and orientation effects in strain-relaxed epitaxial layers remains today as the most common and important application of PSD-based reciprocal space mapping in our laboratory. The growth of strain-relaxed epilayers is motivated by new band-gap and device engineering concepts which continue to call for epitaxial material systems that have 
either strain requirements, or average composition requirements, that do not allow for growth on traditional elemental, or binary-alloy, substrates. Strained-Si, enhancedmobility MOSFETS [11], and strained-layer-superlattice, (InAlGa)As, optoelectronic modulators operating at a wavelength of $1.3 \mu \mathrm{m}$ [12], come to mind as timely examples. In both cases, a strain-relaxed epitaxial buffer layer is used to match the device structure to a commonly available substrate.

Figure 4 shows the (004) rocking curve of a 12-layer, $\operatorname{In}_{\mathbf{x}}(\mathrm{AlGa})_{1-\mathrm{x}} \mathrm{As}$ buffer structure grown on GaAs. The buffer structure is compositionally step-graded from $4 \%$ In to $40 \%$ In and is capped with an $\mathrm{In}_{0.35} \mathrm{Ga}_{0.65}$ As layer, which approximates the target composition of the device structure during development of the buffer. As shown in Figure 4 , ordinary rocking curve analysis of these highly strain-relaxed buffer structures is not useful because lattice strain variations and domain orientation effects are convolved leading to a single broad peak for the multilayer buffer structure. This convolution occurs because of the open-slit detector used in rocking curve analysis. The detector carries out an integration over the surface of the

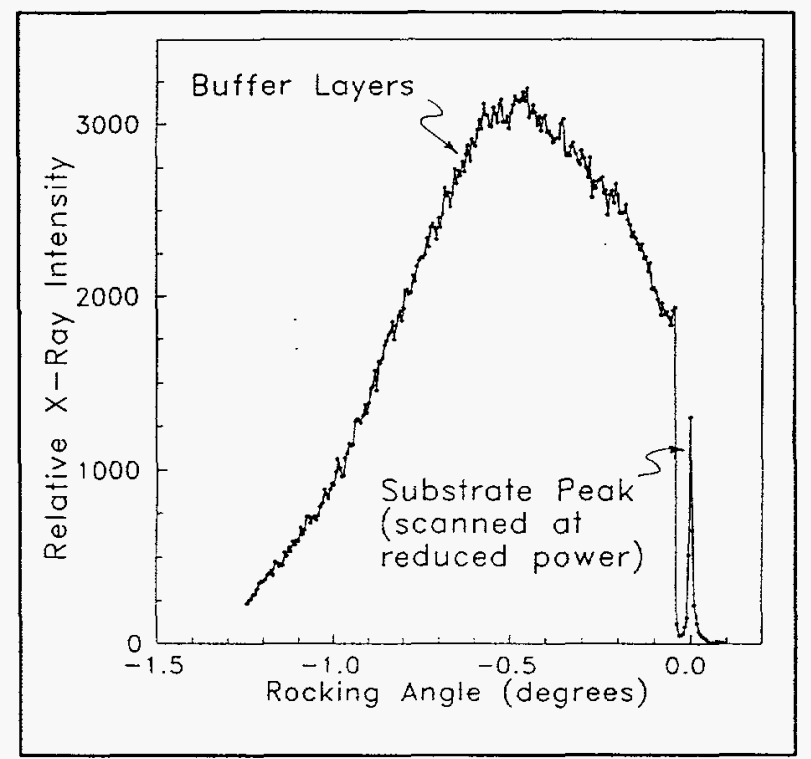

Figure 4. The (004) rocking curve of a highly relaxed, 12-layer, In (AlGa) As $(x=0.04-0.4)$, buffer structure with an $\operatorname{In}_{0.35} \mathrm{Ga}_{0.65}$ As cap. Ewald Sphere at each rocking angle of the scan. This collapses the three-dimensional information of reciprocal space into one dimension, and in the case of strain-relaxed buffers, irretrievably convolves strain and orientation effects.

Figure 5 shows (004) and (224) reciprocal space maps of the same structure. Two key advantages are obtained by making a full reciprocal space analysis using a PSD. First, the loss of information due to convolution can be completely avoided, and the entire structure of the multilayer buffer can be characterized. In contrast to the rocking curve analysis, all the layers making up the buffer-layer structure are clearly resolved in the reciprocal space map. Though the details are beyond the scope of this brief overview, the peak centroid positions are easily analyzed to determine both the lattice strain along [hh0] and [001] directions, and the average tilt of each layer. Using Vegard's law and elasticity theory, the strain in each layer can then be converted to an equivalent alloy composition. Thus, the (004) and (224) reciprocal space maps yield a layer-by-layer profile of the average strain, composition and tilt.

These reciprocal space maps can also give quantitative information about the distribution of tilts, the variation in strain, and in some cases, the size of relaxed domains in each layer. Here, the change in orientation of the buffer peaks, on going from (004) to (224) diffraction conditions, unambiguously identifies tilting of localized domains as the 
source of peak broadening along the peaks' major axes. The increase in peak width with the addition of each buffer layer can be used to determine a depth profile of mosaic spread in the structure. The width of the buffer-layer peaks along their minor axes depends in part upon the distribution of strain in the layer, and in part upon finite crystal size effects. Though not shown in our example, the effect of strain dispersion can be enhanced in order to conclusively separate size effects from variations in layer strain by going to higher order reflections like (335) and (006).

The second major advantage is, of course, the very short data collection time provided by use of the PSD. Because PSDbased reciprocal space mapping is fast, we routinely use reciprocal space mapping to provide crystal growers with structural information during the course of development of new processes and devices. The (004) map in Figure 5(a) was made using a $240 \times 260$ pixel array of measured intensity values. The map was made by stepping through 240 discrete rocking angles, with a counting time of 3 seconds per rocking angle, to give a total scan time of $240 \times 3$ seconds, or 12 minutes. All $2 \theta$ information was collected in parallel at each rocking angle using the $\mathrm{PSD}$. The time required to serially acquire the same data using a mechanically scanned slit, under the assumption of otherwise identical scanning conditions, would be $240 \times 260 \times 3$ seconds, or 52 hours. Using a PSD to acquire the reciprocal space map reduces the data collection time by a factor of 260 !

Figure 5. (a) (004) reciprocal space map of the
buffer structure analyzed in Figure 4 showing the
peak structure which emerges when a two-
dimensional map is made, data collection time for
this map was 12 minutes, (b) (224) reciprocal space
map of the same sample, rotation of the major axes
of the buffer peaks indicates the presence of mosaic.

Figure 5. (a) (004) reciprocal space map of the buffer structure analyzed in Figure 4 showing the peak structure which emerges when a twodimensional map is made, data collection time for map of the same sample, rotation of the major axes of the buffer peaks indicates the presence of mosaic.

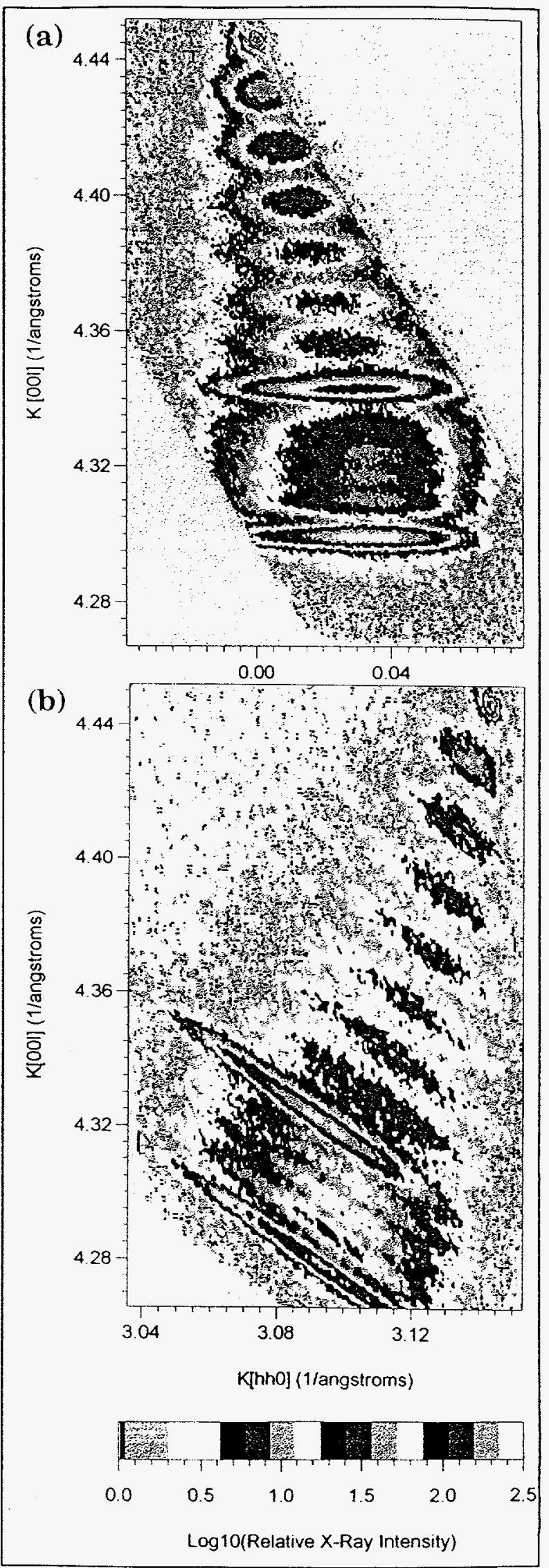




\section{Nondestructive Characterization of the Size, Shape and Orientation of Ordered- Alloy Domains:}

In our laboratory, an emerging application of reciprocal space analysis is to use the reciprocal space image in conjunction with detailed simulations in order to make quantitative determinations of the size, shape and orientation of small, crystalline domains sometimes found in epitaxial layers. In the example presented here, these domains are the nanometer to micrometer-sized orderedalloy domains found in MOVPE-grown $\mathrm{In}_{0.48} \mathrm{Ga}_{0.52} \mathrm{P}$ exhibiting CuPt-type ordering. We have found that reciprocal space mapping using $x$-ray diffraction and a PSD may be used as a complementary or alternative technique to TEM analysis of ordering in compound semiconductors.

CuPt-type ordering of group III atoms occurs in a number of the III-V ternaries when the group III atoms, In and Ga in the present example, partially or fully segregate onto alternating $\{111\}_{<B>}$, group III planes. This doubles the period of the stacking sequence of $\{111\}_{<B>}$ planes and gives rise to half-integer order diffraction spots in reciprocal space. The usual technique for diagnosing the presence of ordering is to make TEM diffraction patterns using thinned, cross-sectional samples in order to observe the half-integer order spots. Provided that a particular type of ordering can be expected, this same task can be accomplished nondestructively, and in a much shorter time, by using PSD-based reciprocal space analysis to map the region of reciprocal space where the intensity due to ordering should appear. Such an analysis for $1 / 2(1,-1,5)$ diffraction planes in $\mathrm{In}_{0.48} \mathrm{Ga}_{0.52} \mathrm{P}$ is shown in Figure 6(a). The presence of a diffraction peak at this location qualitatively indicates the presence of CuPt-type ordering just as in a TEM diffraction pattern.

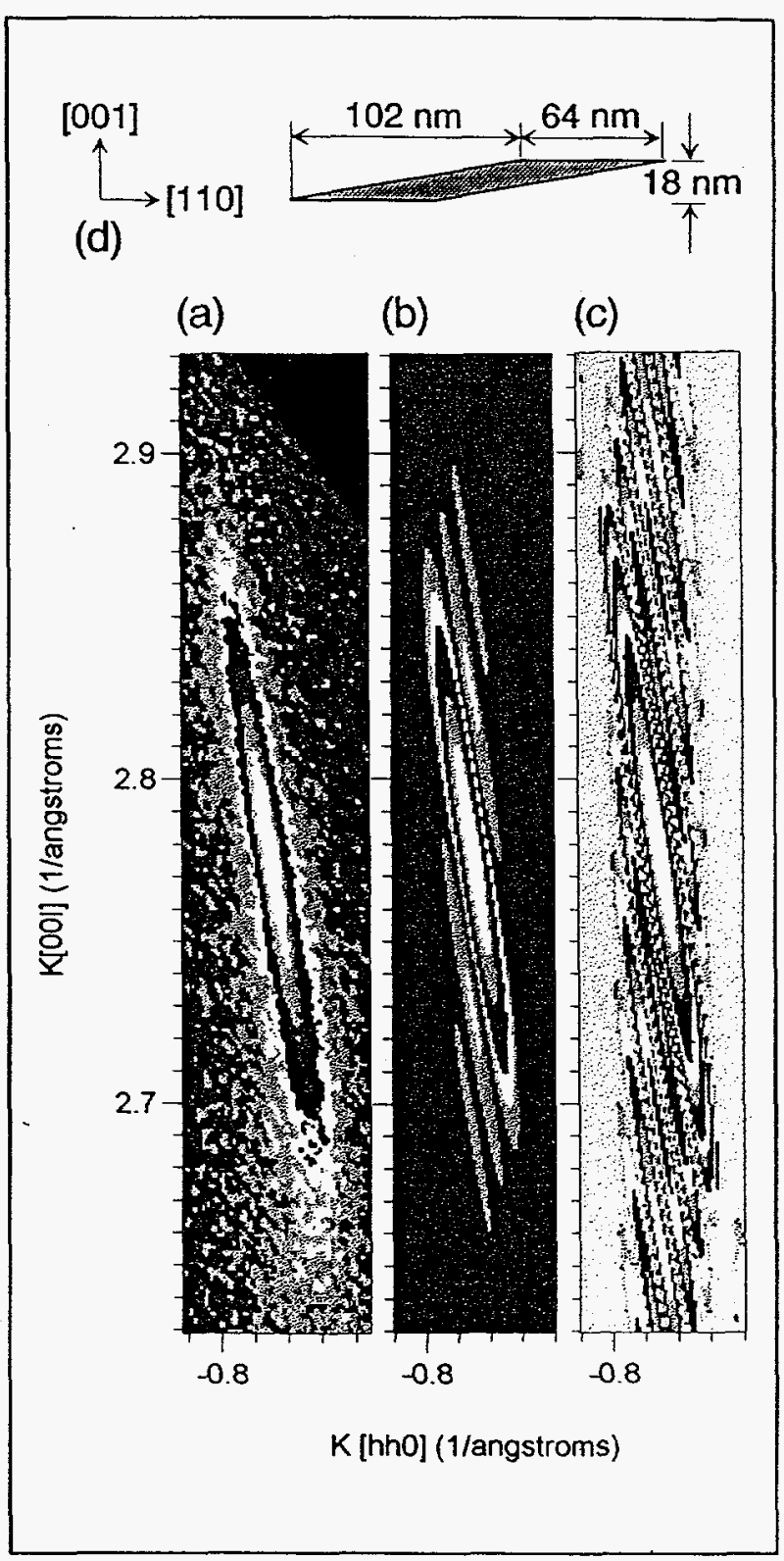

Figure 6. (a) $1 / 2(1,-1,5)$ reciprocal space map of ordered domains in $\mathrm{In}_{0,48} \mathrm{Ga}_{0.52} \mathrm{P}$, (b) kinematical simulation with background added for comparison to experiment, (c) kinematical simulation without background added, (d) ordered-alloy domain geometry used in simulations. 
In addition to just detecting ordering, the size, shape and orientation of the ordered domains can be quantitatively determined by simulating the measured reciprocal space map. As a simple demonstration of this idea, the diffraction pattern of a parallel-piped shaped domain with an average size and orientation like that shown in Figure 6(d) was calculated using the kinematical theory for diffraction from a small crystal as described by Warren [13]. The results of the calculated intensity distribution with and without a uniform background intensity added are shown in Figures 6(b) and 6(c), respectively. The simulated domain size, shape and orientation were adjusted to approximate the FWHM of the measured elliptical intensity distribution along its major and minor axes. The average domain geometry found by PSD-based reciprocal space mapping is in agreement with darkfield TEM images of ordered domains in the same material [14].

\section{Measurement of Void Size and Shape in Porous Anodized Single-Crystals:}

The last example to be presented, the reciprocal space analysis of a porous Si film, continues the theme of quantifying crystal size and shape effects using reciprocal space analysis. Figures 7 (a) and 7 (b) show (004) and (224) reciprocal space maps of porous $\mathrm{Si}$ prepared by anodizing an $\mathrm{n}^{+} \mathrm{Si}$ wafer for 15 minutes at $80 \mathrm{~mA} / \mathrm{cm}^{2}$ using a $50 \%$ solution of $\mathrm{HF}$ in ethanol. This preparation leads to columnar pores oriented normal to the surface along [001] giving a porosity of $43 \%$.

The reciprocal space maps show a number of explicit features which would be difficult to identify using only a rocking curve. In Figures 7 (a) and $7(\mathrm{~b})$, the weaker intensity, uppermost elliptical peak is due to the substrate. The central elliptical peak is due to the single-crystal porous Si layer. Comparison of the relative positions of the porous $\mathrm{Si}$ peak, and the substrate peak, show that the porous $\mathrm{Si}$ is commensurate with the substrate and has a free-standing lattice parameter which clearly differs from that of bulk Si. The tilted streak through the porous Si peak is an artifact that results from the tails in the detector $2 \theta$ response that were described above. The low-intensity vertical tail, or "dagger-like" feature, below the main porous $\mathrm{Si}$ peak is believed to arise due to a distribution of strains in the porous Si layer.

Finally, the low-intensity, horizontal feature oriented parallel to [hh0] is believed to arise from the presence of the vertical pores in the material. The characteristic width of this feature along [hh0], $-0.012 \AA^{-1}$, suggests a real space size in the plane of the porous layer of $-50 \mathrm{~nm}$. TEM analysis of porous $\mathrm{n}^{+}$Si prepared under similar conditions shows pore diameters of $20 \mathrm{~nm}$ [15]. Since the porosity of the present sample is high, the mean spacing of the pores will approach the pore diameter in size. Knowledge of the reciprocal space width of this feature along [001] is resolution-limited by our device and is only known to be less than $-0.0015 \AA^{-1}$. The corresponding real space length of these domains along [001] must be greater than $-420 \mathrm{~nm}$. This in agreement with TEM, which shows that the columns and pores in this type of $\mathrm{n}^{+}$porous $\mathrm{Si}$ extend uninterrupted through the entire thickness of the porous layer. 
Both randomly spaced [001]-oriented pores, and randomly spaced [001]-oriented columns, would give a reciprocal space feature oriented parallel to [hh0]. The choice between either voided pores, or columns of material, is made using further details of the horizontal distribution. Columns of material should yield a feature with a strong central peak which has a width along [hh0] characteristic of the average column diameter, and any fringing features might be expected to have a spacing along [hh0] that is about one-half the base width of the main peak. The spacing of the horizontally oriented lobes of intensity in Figure 7 do not fit this description.

Alternatively, when the Fourier transform is made of a small crystal containing a columnar void oriented along [001], in order to simulate diffraction from (004) or (224) planes, one obtains a strong central peak due to the main crystal, and a series of weak satellite peaks along [hh0] due to void. The spacing, width, and intensity of the satellites depends in detail on the crystal size and shape, the pore size and shape, and the pore position within the crystal. If a number of randomly positioned, [001]-oriented pores are inserted into the crystal, the satellite fine structure due to the voids is expected to degrade into a single pair of subsidiary intensity lobes. The features oriented along [hh0] in Figure 7 are thought to be of this type. The reasoning here is somewhat analogous to the case of ordinary superlattices, where the superlattice's higher order peaks decay away as the

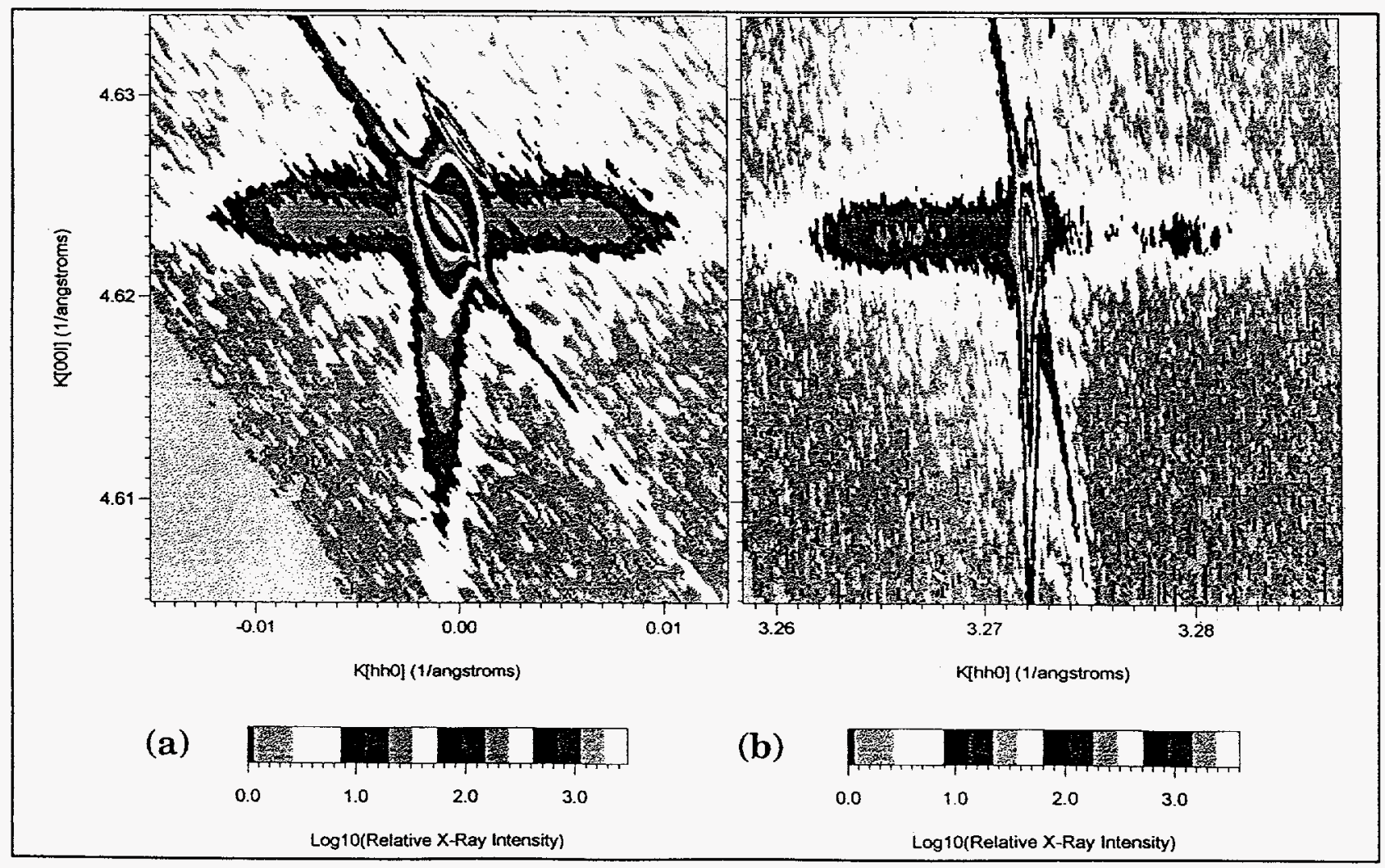

Figure 7. (a) (004) reciprocal space map of a porous Si film prepared by anodization of $\mathrm{n}^{+} \mathrm{Si}$ at $80 \mathrm{~mA} / \mathrm{cm}^{2}$ using 49\% HF in ethanol, (b) (224) reciprocal space map of the same sample, failure of the horizontal intensity distribution to change orientations between (004) and (224) analyses indicates the presence of a crystal size effect -- in this case porosity in the anodized layer. 
sharpness of the layer interfaces degrades, or as the layer periodicity becomes more random. Either effect will eventually eliminate all but the lowest order satellites. We are presently developing a kinematical model of a crystal containing a random array of voids in order to more fully interpret the reciprocal space maps of porous Si layers.

\section{SUMMARY}

Position-sensitive $\mathrm{x}$-ray detection can be used to increase the speed with which reciprocal space can be mapped by approximately two orders of magnitude when compared to serially scanning with an analyzer crystal, or slit, mounted on a triple-axis device. The compromises that are made with the present device, which uses a curved-wire proportional counter as the PSD, are an order magnitude decrease in the $2 \theta$ resolution, and in the worst case, up to two or three orders of magnitude decrease in dynamic range. Future systems are envisioned which will have the high-speed parallelism of our present instrument, and which will approach the performance of a conventional triple-axis instrument in terms of resolution and dynamic range. These systems will use improved x-ray optics optimized for use with PSDs, and advanced PSDs based on solid state technology, to overcome current technical limitations.

The present system has proven to be extremely useful for efficient reciprocal space analysis of a variety of epitaxial materials. The most important use in our laboratory is the routine reciprocal space analysis of strain and mosaic in strain-relaxed multilayers. PSDbased reciprocal space mapping allows us to provide the crystal grower with detailed strain, composition, and orientation profiles derived from complete, two-dimensional, (004) and (224) reciprocal space maps. The profiles for a single sample can be determined in as little as -1-2 hours for structures for which there is a well-established analysis regimen.

Finally, an emerging application of reciprocal space mapping is the detailed characterization of crystal size, shape and orientation effects in novel or imperfect epitaxial structures. The analysis of ordered domains in III-V compound semiconductors, and voids in porous Si films, are examples where the real-space microstructure of epitaxial materials can be examined through reciprocal space analysis.

\section{ACKNOWLEDGMENTS}

We thank David M. Follstaedt for several useful discussions of ordering and phase separation phenomena in InGaP alloys. This work was supported by the U.S. Department of Energy under Contract DE-AC04-94AL85000. 


\section{REFERENCES}

[1] H. Heinke, M. O. Möller, D. Hommel and G. Landwehr, J. Crystal Growth 135 (1994) 41.

[2] P. Kidd and P. F. Fewster, Proceedings of the 1993 Fall Meeting of the Materials Research Society, Symposium B, Boston, MA, November 19 - December 3, 1993, (to be published).

[3] P. van der Sluis, M. J. Verheijen and J. Haisma, Appl. Phys. Lett. 64 (1994) 3605.

[4] S. T. Horng, M. S. Goorsky, J. H. Madok and N. M. Haegel, J. Appl. Phys. 76 (1994) 2066.

[5] R. J. Matyi, M. R. Melloch and J. M. Woodall, Appl. Phys. Lett. 60 (1992) 2642.

[6] A. McL. Mathieson, Acta Cryst. A38 (1982) 378.

[7] L. R. Thompson, G. J. Collins, B. L. Doyle and J. A. Knapp, J. Appl. Phys. 70 (1991) 4760.

[8] S. T. Picraux, B. L. Doyle and J. Y. Tsao, Chapter 3, Semiconductors and Semimetals 33 (AT\&T, 1991) 139-222.

[9] M. Braun GmbH, Technical data for detector Type-OED 50/R250 (1987).

[10] M. A. Pick, K. Bickmann, E. Pofahl, K. Zwoll and H. Wenzl, J. Appl. Cryst. 10 (1977) 450.

[11] J. Welser, J. L. Hoyt and J. F. Gibbons, IEEE Electron Device Lett. 15 (1994) 100.

[12] I. J. Fritz, J. A. Olsen, A. J. Howard, T. M. Brennan, B. E. Hammons and G. A. Vawter, IEEE J. Quantum Electron. 30 (1994) 452.

[13] B. E. Warren, Chapter 3, X-Ray Diffraction (Addison-Wesley, 1969) 27-40.

[14] D. M. Follstaedt, R. P. Schneider, Jr., and E. D. Jones, Mat. Res. Soc. Symp. Proc. 326 (1994) 61.

[15] D. L. Smith and S. D. Collins, J. Appl. Phys. 71 (1992) R1.

\section{DISCLAIMER}

This report was prepared as an account of work sponsored by an agency of the United States Government. Neither the United States Government nor any agency thereof, nor any of their employees, makes any warranty, express or implied, or assumes any legal liability or responsibility for the accuracy, completeness, or usefulness of any information, apparatus, product, or process disclosed, or represents that its use would not infringe privately owned rights. Reference herein to any specific commercial product, process, or service by trade name, trademark, manufacturer, or otherwise does not necessarily constitute or imply its endorsement, recommendation, or favoring by the United States Government or any agency thereof. The views and opinions of authors expressed herein do not necessarily state or reflect those of the United States Government or any agency thereof. 